\title{
Implementation of Deep Dictionary Learning and Coding Network for Plant Disease Detection in Agricultural Field
}

\author{
Snehal A. Lale ${ }^{1}$, Dr. V. K. Shandilya ${ }^{2}$
}

${ }^{1}$ PG Scholar, Department of Computer Science of Engineering, Sipna COET Amravati, Maharashtra, India ${ }^{2}$ Professor and Head, Department of Computer Science of Engineering, Sipna COET Amravati, Maharashtra, India

\section{Article Info}

Volume 7, Issue 3

Page Number: 547-553

Publication Issue :

May-June-2021

\section{Article History}

Accepted : 01 June 2021

Published : 10 June 2021

\section{ABSTRACT}

A web based online application is built to detect the diseases present on plant leaves. This will help farmer/end user to find out which diseases are present on that leaves. In older times, this task requires much time \& resources to do the detection. A approach has of DDLCN has been taken in this project which will help to find out the best match features. In this approach. I have taken up to 3 layers of DDLCN which will filter the most match features of the diseases present on plant leaves. The proposed DDLCN combines the two things that is deep learning \& dictionary learning. Deep learning which the branch of AI. It has a structures inspired by the human brain. It has the artificial neural networks which helps to train the data. Now taking about the dictionary learning which has a literal meaning having large number of data set. Dictionary learning is also called as sparse representation which will help to arrange the data in proper manner and without wasting the storage. Because it will only count the non zeros numbers present in the sparse matrix.

Keywords: DDLCN, deep learning, technique, Network, Sparse representation

\section{INTRODUCTION}

We have develop an online web based application which will help to find out the diseases present on the plant leaves. The detection of disease at early stage will comes out to be less spread of disease at other plants. This application will help farmer /end user to find out disease on their plant leaves. The important asset of sparse representation is that the complicated signal can be represented in a short manner enabling the following classification procedure to adopt a simpler classifier. In latter times, server based and mobile based approach for disease identification has been employed for disease identification. Several factors of these technologies being big resolution camera, high performance processing and ample of built in accessories are the added advantages resulting in automatic disease recognition. [7] Here, I have used SIFT algorithm which will help to extract the features present on the 
plant leaves. Features means it will recognize the pixels from the image and does processing on it \& find the affected pixels. Different types of algorithms are used to perform the detection of the diseases.

\section{LITRATURE REVIEW}

It is worth noting that sparse-inducing resolve and sparse representation have been demonstrated to be extraordinarily powerful for representing complicated signals in recent works. Signals such as audios, videos and images admit naturally sparse representations, while the focal idea of sparse representation is delineating the original chaotic signals to their corresponding concise representations with a regularized or uniform style. These sparse representations have an intimate relationship due to their over-complete dictionaries. [6] The classification and detection techniques that can be used for plant leaf disease classification. Here preprocessing is done before feature extraction. RGB images are transformed into white and then converted into grey level image to extract the image of vein from each leaf. Then salient Morphological functions are applied on the image. Then the image is converted into binary image. After that if binary pixel value is 0 it's converted to corresponding RGB image value. Finally by using Pearson correlation and Dominating feature set and Naïve Bayesian classifier disease is detected. [1]

They have put forward for a technique that can be used for detecting paddy plant disease by comparing it with 100 healthy images and 100 sample of disease 1 and another 100 sample of disease2. It's not adequate enough to detect disease or classify it training data is not linearly separable. The feature selection technique has speedy execution speed and higher classification success rate because it does not suffer from the local minima problems inherent in the nonlinear modeling techniques typically used in forward selection and backward elimination. [8]

\section{ANALYSIS OF PROBLEM}

Farmer's economic growth depends on the quality of the products that they mass-produce, which relies on the plant's growth and the yield they get. Plants are highly liable to diseases that affect the growth of the plant which in turn affects the ecology of the farmer. Manual detection of plant disease is a tedious job. Plant disease recognition by visual way is more laborious task and can be done only in limited areas. More digital approaches are coming in recent years to detect the disease on the plant.

The old times method for plant disease detection is simply naked eye examination by experts through which identification and detection of plant diseases is done. For doing so, a large team of experts as well as continuous monitoring of plant is require/swed, which costs very high when we do with large farms. At the same time, in some countries, farmers do not have proper provision or even idea that they can contact to experts. Due to which visiting experts even cost high as well as time consuming too. In such conditions, the recommended technique proves to be advantageous in monitoring large fields of crops. Automatic detection of the diseases by just seeing the signs on the plant leaves makes it easier as well as cheaper. This also supports machine vision to provide image based automatic process control, inspection, and robot guidance [11] [12] [13]

\section{WORKING}

Feature extraction plays a key role for identification of an object. In many approach of image processing feature extraction is used. Color, texture, morphology, edges etc. are the features which can be taken into consideration in plant disease detection. 
Now the implementation goes like this. First the admin will register him/her \& then login to the portal. After this admin will register the expert \& provide him/her the login id and password. Now the work of expert will goes like he/she will register the crop wise diseases and that registration means it will follow all the processing that's is the image will go through first image preprocessing which will be done by the Haar wavelet transform algorithm. It will compress the image \& convert it into grey scale After that Feature extraction will be perform on that data by SIFT algorithm which extract the features of plant leaves \& store by using sparse representation on the server.

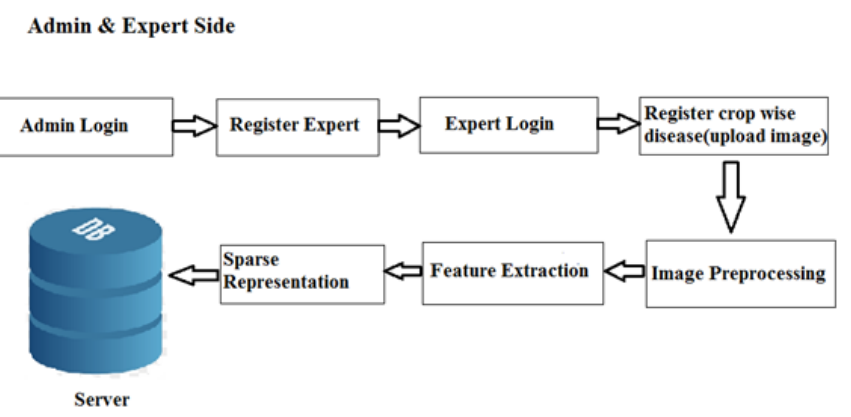

Fig. 1 Flow chart of admin \& expert side

The below flow chart shows the working done at farmer/end user side. Farmer/end user will register them on the web application. After registration he/she will upload the image for disease detection on plant leave And on that uploaded image preprocessing is perform which will be done by again Haar wavelet transform which will compress the image \& convert it into gray scale. The wavelet means these are small waves which are in the form of square shaped signal used to process the data. Now the converted image will undergoes the feature extraction which will be done by the Scale Invariant Transform. This algorithm will extract the features of image \& finally this extracted features will be given to the DDLCN network which does the comparison of upcoming data with existing data to find the exact result for the detection.

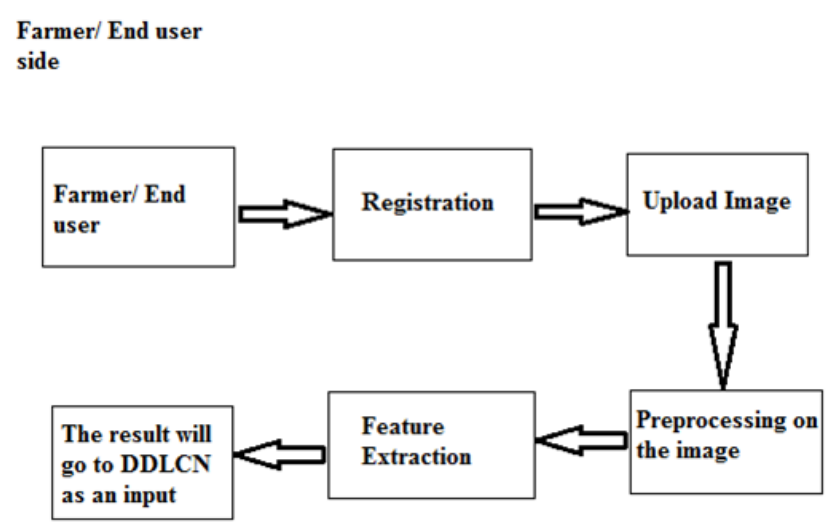

Fig. 2 Flow chart of farmer/end user

\section{ALGORITHMS}

\section{Haar Wavelet Algorithm}

The Haar sequence was put forward in 1909 by Alfréd Haar. Haar used these aims to provide an example of an orthonormal system for the space of square-integral functions on the unit interval $[0,1]$ The research of wavelets, and even the term "wavelet", did not come until much later. As a special case of the Daubechies wavelet, the Haar wavelet is called as Db1. In mathematics, the Haar wavelet is a cycle of rescaled "square-shaped" functions which jointly form a wavelet family or root. Wavelet analysis is analogous to Fourier analysis in that it allows a target function over an intermission to be represented in terms of an orthonormal basis. The Haar sequence is now recognized as the first called wavelet basis. The Haar wavelet is also the easiest possible wavelet. Wavelets are a kind of a functions used to restrain a given function in both space and scaling. A family of wavelets can be constructed from a function, sometimes called as a "mother wavelet," which is confined in a limited interval. "Daughter wavelets" are then created by translation () and contraction (). 


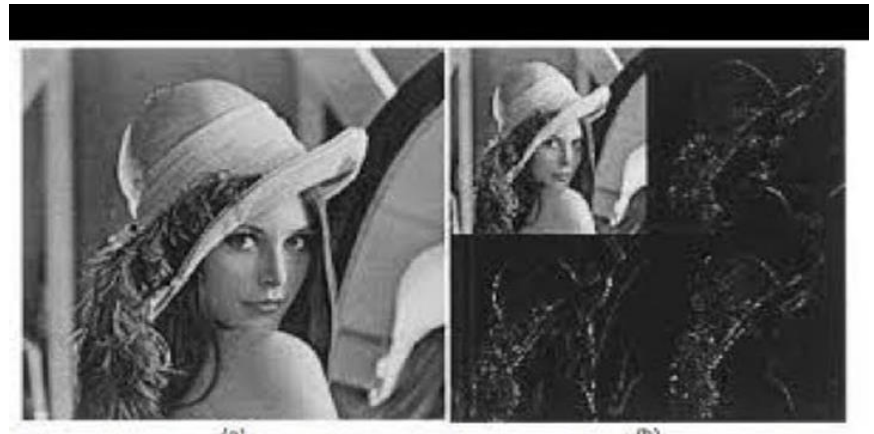

\section{Haar Wavelet Transform}

Fig.3 Processing of image

\section{SIFT}

It is Scale invariant Transform Algorithm. It is a used for detecting component of an image. For every such point, it also provides a set of features that characterize/describe a tiny image region around the point. These features are invariant to rotation and scale. To determine correlation correctly, we need some features characterizing a salient point.

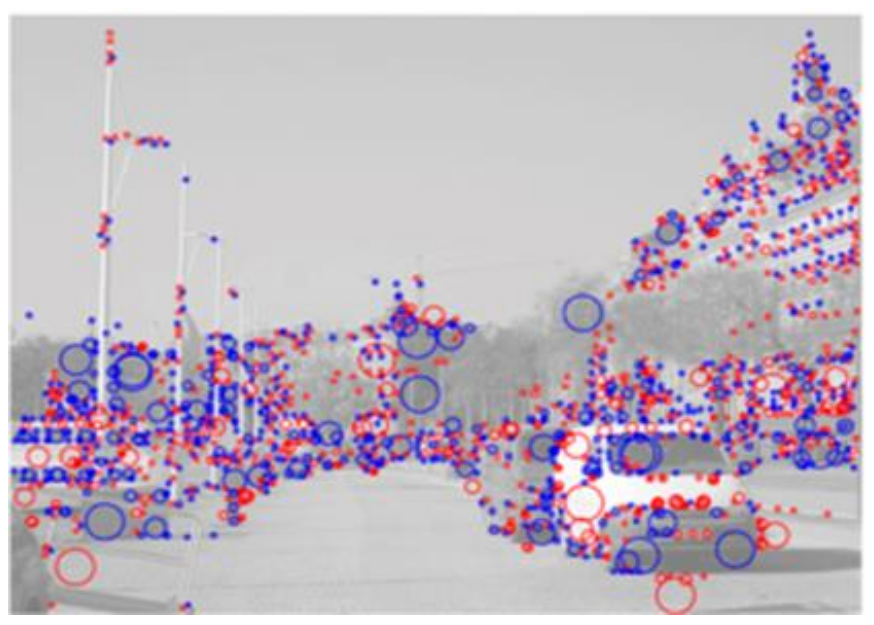

Fig.4 Pointing out the features image

\section{Sparse Representation}

Sparse representation is an approach in which the linear combination of a few atoms from an overcomplete dictionary can be used to represent a signal. Sparse representations of a signal are easier to narrate because they're short and highlight the essential features. Sparse Representation is obtain by using sparse matrix. Sparse Matrix means it has the majority of elements equal to zero. Sparse Matrix representation can done in two ways Array, link list. Nowadays, sparse coding has appeared as one promising technique in a wide range of applications, including image recovery, analysis, and classification. In classic sparse modeling problem, the sparse coding aims at finding sparse representation of input data under an adaptive dictionary. There has been an abundant literature on its analysis and algorithms. [14] The sparse dictionary learning technique has been successfully used in various applications specifically for classification.

\section{Deep Dictionary Learning}

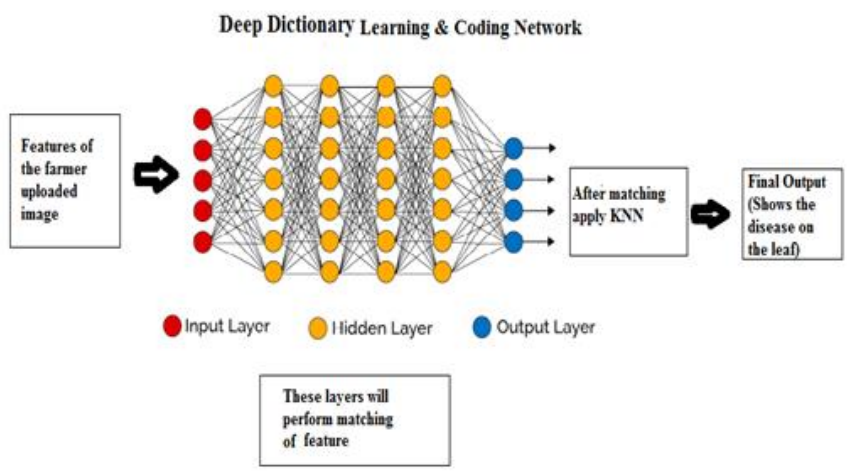

Fig. 5 Deep Dictionary learning \& Coding network

Dictionary learning is learning a set of atoms so that a given image can be well resemble by a sparse linear combination of these learned atoms. Dictionary learning also known sparse representation or sparse coding, represents a signal with a straight merger of a few elements/atoms from a dictionary matrix containing several prototype signal atoms. Deep learning methods aim at extracting deep semantic feature depicts via a deep network. Deep learning is a type of machine learning motivated by the structure of human brain. In deep learning the features are picked out by the neural network without human 
invention but off course that kind of independence comes at the cost of much higher volume of the data to train a machine. Neural network is train to identify the images.

\section{RESULT}

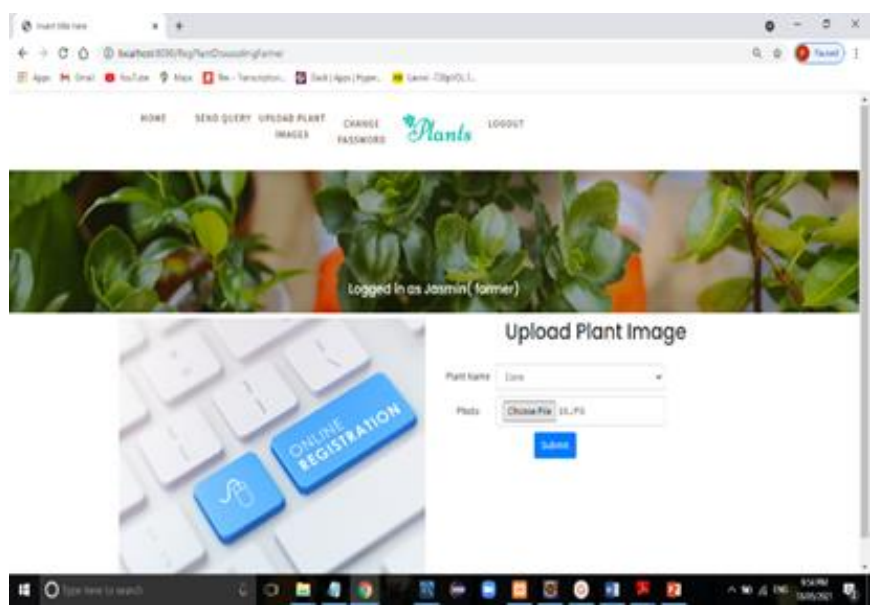

Fig. 6. Upload the image for testing

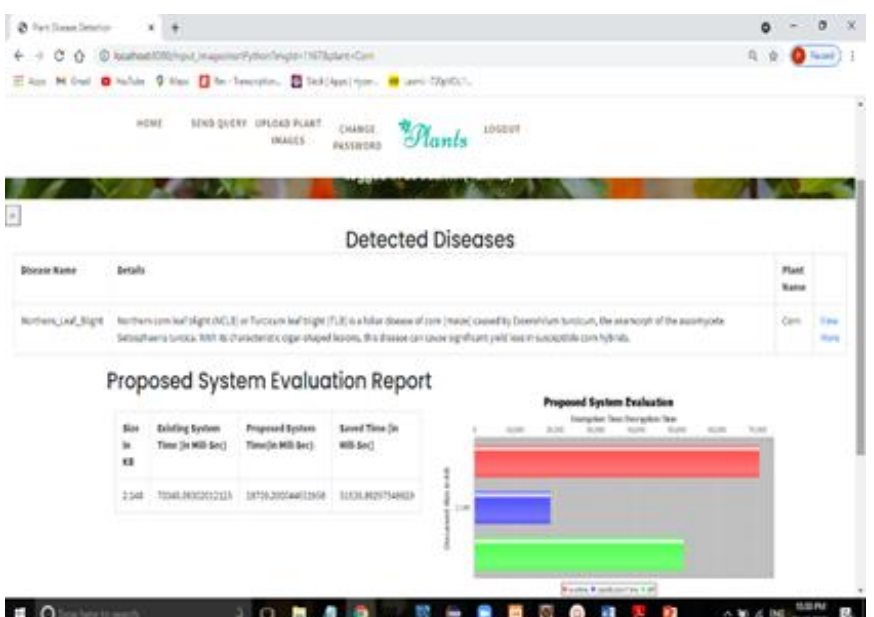

Fig.7. Final result with comparative Analysis

\section{Comparative Analysis}

\section{Existing System}

The Histogram of Oriented Gradients (HOG) is a feature descriptor used in computer vision and image processing applications for the purpose of the object detection. It is a technique that counts events of gradient orientation in a specific portion of an image or region of interest. Steps for execution, converts the input image to black and white. HOG only considers the changes between the light and dark areas in the image. It ignores the color information. That's why it converts colored image into the black and white image. Now after the step 1, it looks for the gradient in each pixel. A gradient is a direction from the lighter area to the darker area. It repeats the process for the entire pixels of a black and white image and draws the gradient image of it.

\section{Proposed System}

A novel deep dictionary learning DDLCN framework that combines the advantages of both dictionary learning and deep learning methods. This introduce a novel compound dictionary learning and coding layer, which can substitute the convolutional layer in the standard deep learning architectures. Along with DDLCN proposed DDLCN for 2 layers and 3 layers Specifically, DDLCN-2 uses two compound dictionary learning and coding layers: the first layer aims to learn a dictionary to represent the input image and the second layer target to learn a dictionary to represent the activated atoms in the first layer. For a given input image, we use both the first and second dictionaries to learn the coding representation and then concatenate both of them to obtain the final coding representation. For a deeper version, i.e., DDLCN-3, which uses three compound dictionary learning and coding layers, the first layer aims to learn a dictionary to represent input image, then the second layer targets to learn a dictionary to represent the activated atoms of the first layer, and, finally, the third layer learns another dictionary to represent the activated atoms of the second layer.

Comparison between the existing \& the proposed one. 
Table 1. Evolution Table

\begin{tabular}{|l|l|l|l|}
\hline $\begin{array}{c}\text { Size } \\
\text { in } \\
\text { KB }\end{array}$ & $\begin{array}{l}\text { Existing System } \\
\text { Time (in Mili- } \\
\text { Sec) }\end{array}$ & $\begin{array}{c}\text { Proposed } \\
\text { System Time(in } \\
\text { Mili-Sec) }\end{array}$ & $\begin{array}{l}\text { Saved Time (in } \\
\text { Mili-Sec) }\end{array}$ \\
\hline \begin{tabular}{l|l|l|l|}
2.24 \\
2
\end{tabular} & $\begin{array}{l}50825.98362634 \\
3485\end{array}$ & $\begin{array}{l}12448.99172782 \\
898\end{array}$ & $\begin{array}{l}38376.99189851 \\
4505\end{array}$ \\
\hline $\begin{array}{l}2.24 \\
2\end{array}$ & $\begin{array}{l}54661.81000811 \\
555\end{array}$ & $\begin{array}{l}16087.93121528 \\
6255\end{array}$ & $\begin{array}{l}38573.87879282 \\
9295\end{array}$ \\
\hline $\begin{array}{l}2.41 \\
6\end{array}$ & $\begin{array}{l}45135.56304947 \\
211\end{array}$ & $\begin{array}{l}12623.93975448 \\
6084\end{array}$ & $\begin{array}{l}32511.62329498 \\
6028\end{array}$ \\
\hline $\begin{array}{l}2.59 \\
6\end{array}$ & $\begin{array}{l}46123.21781849 \\
861\end{array}$ & $\begin{array}{l}12676.90512275 \\
6958\end{array}$ & $\begin{array}{l}33446.31269574 \\
165\end{array}$ \\
\hline $\begin{array}{l}2.34 \\
6\end{array}$ & $\begin{array}{l}49745.27845275 \\
536\end{array}$ & $\begin{array}{l}12764.69607353 \\
2104\end{array}$ & $\begin{array}{l}36980.58237922 \\
3255\end{array}$ \\
\hline
\end{tabular}

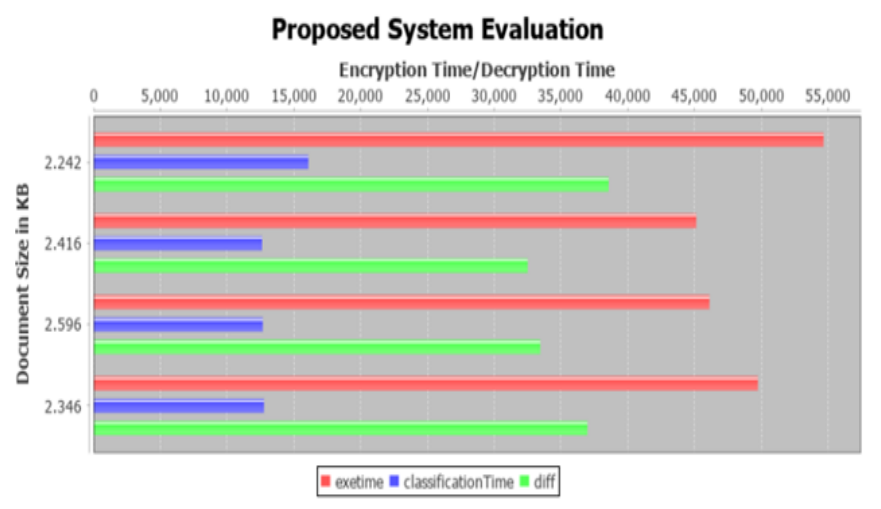

Fig. 9. Evolution Graph

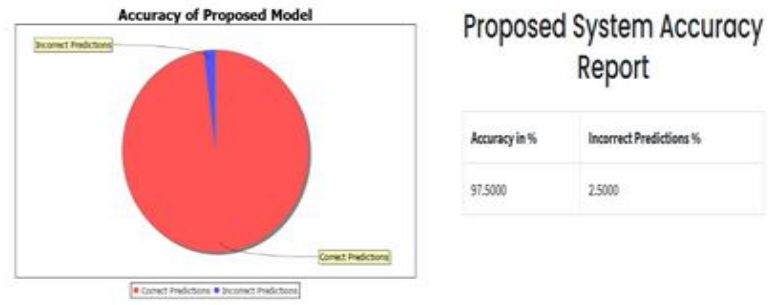

Fig. 10. Accuracy Chart

\section{CONCLUSION}

In this paper, we proposed sparse coding based plant image classification system. We compared our proposed system with existing feature extraction algorithm that is Histogram of Oriented Gradients with KNN. In our proposed system, we have used sparse coding for feature extraction of input image. Sparse coding is used to extract most prominent features only from image. Therefore we require less execution time as compared to other classification techniques. With the help of sparse coding, we reduced no of image features as compared to HOG feature extraction algorithm. As we are extracting only important features of the image, time require to process that features using KNN is reduced and hence we can conclude that our proposed system is faster than HOG -KNN Classification technique.

\section{FUTURE SCOPE}

In this paper we have reduced the classification time as compared to existing $\mathrm{HOG}-\mathrm{KNN}$ classification technique to classify plant image into plant diseases appropriately. In this system we are performing classification to predict plant diseases; in future we can add plant classification from input image in addition to disease classification. In future we will try to give the application dynamic inputs so that at run time the application will be able to detect the disease. And one more thing is that in upcoming time another approach will forward to give more accurate result for plant leaves disease.

\section{REFERENCES}

[1]. Dhiman Mondal, Dipak Kumar Kole, Aruna Chakraborty, D. Dutta Majumder(2015) "Detection and Classification Technique of Yellow Vein Mosaic Virus Disease in Okra Leaf Images using Leaf Vein Extraction and Naive Bayesian Classifier", International Conference on Soft Computing Techniques and Implementations- (ICSCTI) Department of ECE, FET, MRIU, Faridabad, India, Oct 8- 10, 2015.

[2]. Pranjali B. Padol, Prof. Anjila Yadav (2016), "SVM Classifier Based Grape Leaf Disease Detection" Conference on Advance n Signal Processing (CAPs) Cummins College Of Engineering for Woman Pune, June 9- 
[3]. Zarreen Naowal Reza, Faiza Nuzhat, Nuzhat Ashraf Mahsa, Md.Haider Ali(2016) "Detecting jute plant disease using image processing and machine learning" 3rd International Conference on Electrical Engineering and Information Communication Technology (ICEEICT).

[4]. Tejoindhi M.R, Nanjesh B.R, JagadeeshGujanuru Math, AshwinGeetD'sa(2016) “Plant Disease Analysis using Histogram Matching Based on Bhattacharya's Distance Calculation" International Conference on Electrical, Electronic and Optimization Technique (ICEEOT)

[5]. M S Arya, K Anjali, Divya Unni“'Detection of unhealthy plant leaves using image processing and genetic algorithm with Arduino" (2018) International Conference on Power, Signals, Control, And Computation (EPSCICON).

[6]. Hao Tang, Hong Liu ,Wei Xiao and Nicu sebe . "When Dictionary Learning Meets Deep Learning: Deep Dictionary Learning And Coding Network for Image Recognition with Limited Data"(2020). IEEE TRANSACTIONS ON NEURAL NETWORKS AND LEARNING SYSTEMS

[7]. Shima Ramesh, Mr. Ramachandra Hebbar, Niveditha M, Pooja R,Prasad Bhat N, Shashank N , Mr. P V Vinod, "Plant Disease Detection using Machine Learning", 2018 International Conference on Design Innovations for 3Cs Compute Communicate Control. Conference Paper · April 2018.

[8]. Kranti Tambe, Dakshata Lohakare, Manjusha Shinde, Prof. Meghana M. Deshpande, "Detection of Plant Leaf Disease Using Machine Learning" International Research Journal of Modernization in Engineering Technology \& Science, Volume 02/Issue:07/July 2020
[9]. Sergio Matiz and Kenneth E. Barner, "Label Consistent Recursive Least Square Dictionary Learning For Image Classification" National Science Foundation 2016 IEEE.

[10]. Jing Ling, Zhenzhong Chen, Feng Wu, "ClassOriented Discriminative Dictionary Learning for Image Classification" IEEE Transactions on Circuits and Systems for Video Technology.

[11]. Dhaygude Sanjay B, Kumbhar Nitin P. Agricultural plant leafdisease detection using image processing. Int J Adv Res ElectrElectron Instrum Eng 2013;2(1).

[12]. Arivazhagan S, Newlin Shebiah R, Ananthi S, Vishnu VarthiniS. Detection of unhealthy region of plant leaves andclassification of plant leaf diseases using texture features.Agric Eng Int CIGR 2013;15(1):211-7.

[13]. Kulkarni Anand H, Ashwin Patil RK. Applying imageprocessing technique to detect plant diseases. Int J Mod EngRes 2012;2(5):3661-4.

[14]. Yuhui Quan, Yong Xu, Yuping Sun ,Yan Huang and Hui Ji, "Sparse Coding for Classification via Discrimination Ensemble"Computer Vision Foundation IEEE Xplore.

\section{Cite this article as :}

Snehal A. Lale, Dr. V. K. Shandilya, "Implementation of Deep Dictionary Learning and Coding Network for Plant Disease Detection in Agricultural Field", International Journal of Scientific Research in Computer Science, Engineering and Information Technology (IJSRCSEIT), ISSN : 2456-3307, Volume 7, Issue 3, pp.547-553, May-June-2021. Available at doi : https://doi.org/10.32628/CSEIT2173116 Journal URL : https://ijsrcseit.com/CSEIT2173116 